\title{
Influence of Microstructure-Evolution Changes on the Dielectric Properties of Strontium Titanate Prepared via Mechanical Alloying
}

\author{
Mutia Suhaibah Abdullah ${ }^{1 *}$, Dayang Nur Fazliana Abdul Halim¹, Jumiah Hassan ${ }^{1,2}$, Mansor Hashim ${ }^{1}$, Alex \\ See $^{2}$, Suriati Paiman ${ }^{1,2}$ and Rosli Hussin ${ }^{3}$ \\ ${ }^{1}$ Materials Synthesis and Characterization Laboratory (MSCL), Institute of Advanced Technology (ITMA), University Putra \\ Malaysia, 43400 UPM Serdang, Selangor, Malaysia.

\footnotetext{
${ }^{2}$ Department of Physics, Faculty of Science, University Putra Malaysia, 43400 UPM Serdang, Selangor, Malaysia

3 Department of Physics, Faculty of Science, Universiti Technologi Malaysia, 81310 Skudai, Johor, Malaysia
}

*Corresponding author's email: mutiasuhaibah [AT] gmail.com

\begin{abstract}
SrTiO}_{3}$ is a dielectric material of considerable interest. However, the relationships between microstructure and dielectric properties have not been studied in detail. Hence, we have undertaken an extensive experimental work to study the evolution of the dielectric properties against morphological changes of $\mathrm{SrTiO}_{3} . \mathrm{SrTiO}_{3}$ was prepared using the mechanical alloying method and samples with nano-sized starting powder were obtained. The milled powder was pressed into pellets and sintered at various temperatures ranging from $500{ }^{\circ} \mathrm{C}$ to $1400{ }^{\circ} \mathrm{C} . \mathrm{XRD}$ studies showed that these ceramics completely formed a perovskite phase at $900{ }^{\circ} \mathrm{C}$. FeSEM studies show the presence of small grain sizes ranging from 120 to $600 \mathrm{~nm}$. Dielectric constant ( $\left.8 r^{\prime}\right)$ and dielectric loss tangent (tan $\delta$ ) were measured as a function of frequency and correlated with the microstructure. $\varepsilon_{r}$ ' and tan $\delta$ against frequency show a falling trend at the lower frequency region due to the changing interfacial effect. For sintering temperatures $1000{ }^{\circ} \mathrm{C}$ to $1100{ }^{\circ} \mathrm{C}$, grain size and XRD peak data show a significant increase with the corresponding increase in $\mathrm{r}^{\prime}$, suggesting a sudden increase in the polarizability due to significant increase in crystallinity. With increasing sintering temperature, $\varepsilon_{r}$ ' increased proportionally with XRD peak and grain size increases, further confirming polarizability and crystallinity relationship. tan $\delta$ correlation with microstructure does not have a common trend.
\end{abstract}

Keywords- Dielectric properties, evolution, strontium titanate, microstructure

\section{INTRODUCTION}

A lot of researches and attempts were conducted to find ingenious and readily available dielectric materials that could yield predictable and controllable permittivity values with very low dielectric loss has always proved to be positive and successful. Quite a number of materials with high dielectric constant have been reported to date [1-2]. Perovskite-type oxides are some of the materials with high dielectric constant. The ability of the simple perovskite structure $\left(\mathrm{ABO}_{3}\right)$ to give rise to intergrowth structures, oxygen deficient structures and ordered perovskite structures are well known [3-6]. Among the wider family of perovskite-type oxides, $\mathrm{SrTiO}_{3}$ is a representative member of this family; it displays a cubic structure. $\mathrm{SrTiO}_{3}$ as a ceramic material with high dielectric constant $\left(\varepsilon_{\mathrm{r}}{ }^{\prime}\right)$, low dielectric loss tangent (tan $\delta$ ), large polarization and good insulating properties is a potential candidate in electronic industry. Dielectric properties and their voltage dependence are expected to depend strongly on the microstructural parameters of ceramics such as grain size, mechanical stress and porosity [7].

Owing to the need of modern electronic industry, the synthesis methods are used to control the morphology and grain size of $\mathrm{SrTiO}_{3}$. Among these methods, mechanical alloying (MA) is one of the methods that are often used to synthesize $\mathrm{SrTiO}_{3}$ and can effectively control the morphology and grain size of the products [8]. MA is a solid-state powder processing technique involving repeated welding, fracturing, and rewelding of powder particles in a high-energy ball mill [9]. It is also an economical process with important technical advantages. The usefulness of this method has been well documented in the literature and excellent reviews and monographs are available dealing with the different aspects $[9$, $10]$.

Properties of dielectric materials are affected by their microstructures, especially by grain size, porosity and density, which in turn, are strongly influenced by sintering temperature. The effect of sintering temperature variations on the 
physical properties and microstructure of the final product has always been a matter of great importance in ceramic literature since several decades ago [11-14]. Uncovering the relationship between structure and dielectric properties is important for the eventual rational design of new dielectric materials. Most of the works carried out on $\mathrm{SrTiO}_{3} \mathrm{system}$ basically cover the composition of $\mathrm{SrTiO}_{3}$ towards their electrical properties, effect of doping and their dielectric and microstructure relationship at higher sintering temperature. To the best of our knowledge, not much work was done on tracking the evolution of $\mathrm{SrTiO}_{3}$ at low sintering temperature until they evolve to their final microstructure at the final sintering temperature. Reports on microstructure-property evolution study of $\mathrm{SrTiO}_{3}$ which endeavor to correlate the evolving microstructure with the dielectric properties have not been given in details. In order to obtain greater clarification on this aspect; an attempt is made in this work to synthesize $\mathrm{SrTiO}_{3}$ powders by the mechanical alloying method which is followed by compacted-sample sintering in a series of ascending temperatures.

\section{EXPERIMENTAL}

SrTiO3 samples were prepared using mechanical alloying method. $\mathrm{SrCO}_{3}$ and anatase- $\mathrm{TiO}_{2}$ were employed as the starting powders. The starting powders were weighed in stoichiometric proportions and mixed homogeneously by the high energy ball mill (SPEX SamplePrep 8000 Mixer/Mill) for 12 hours. The ball-to-powder weight ratio was 10:1. The powders were mixed with polyvinyl alcohol (PVA) as a binder and zinc stearate as a lubricant and then pressed into pellets. Nine samples were each finally sintered at nine different temperatures $500^{\circ} \mathrm{C}-1400^{\circ} \mathrm{C}$ at $100^{\circ} \mathrm{C}$ intervals for 10 hours in an ambient atmosphere. The density was obtained using Electronic Densimeter MD300S which adopts Archimedes principle.

Phase identification of the synthesized pellet form was performed using X-ray diffraction method. The XRD measurements were carried out with $\mathrm{CuK}_{\alpha}$ radiation operating at $45 \mathrm{kV}, 40 \mathrm{~mA}$ at room temperature using Philips Panalytical X-Ray Diffractometer, equipped with diffraction software analysis. Diffraction patterns were collected in the range from 10 to $90 \mathrm{o}$, in steps of $0.03 \mathrm{o}$. Identification of the diffraction peaks of the XRD patterns was carried out by using the JCPDS database.

A Field Emission Scanning Electron Microscope (FESEM, FEI NOVA NanoSEM 230) operating at $10 \mathrm{kV}$, and transmission electron microscope. (LEO 912AB Energy Filter TEM), operating at $20 \mathrm{kV}$ were employed to observe the morphology of products. The distribution of grain sizes were obtained by taking at least 200 different grain images for each sample and estimating the mean diameters of each individual grain.

The dielectric measurements were performed over the frequency range of $40 \mathrm{~Hz}$ to $10 \mathrm{MHz}$ at room temperature using Agilent 4294A impedance analyzer.

\section{RESULTS AND DISCUSSIONS}

\subsection{Structural Analysis}

Figure 1 reveals the TEM micrograph of as-milled powder after 12 hours of milling. It shows that as-milled particles are essentially of spherical shape which favours ceramic processing with size from $60 \mathrm{~nm}$ to $120 \mathrm{~nm}$ with an average around $80 \mathrm{~nm}$.

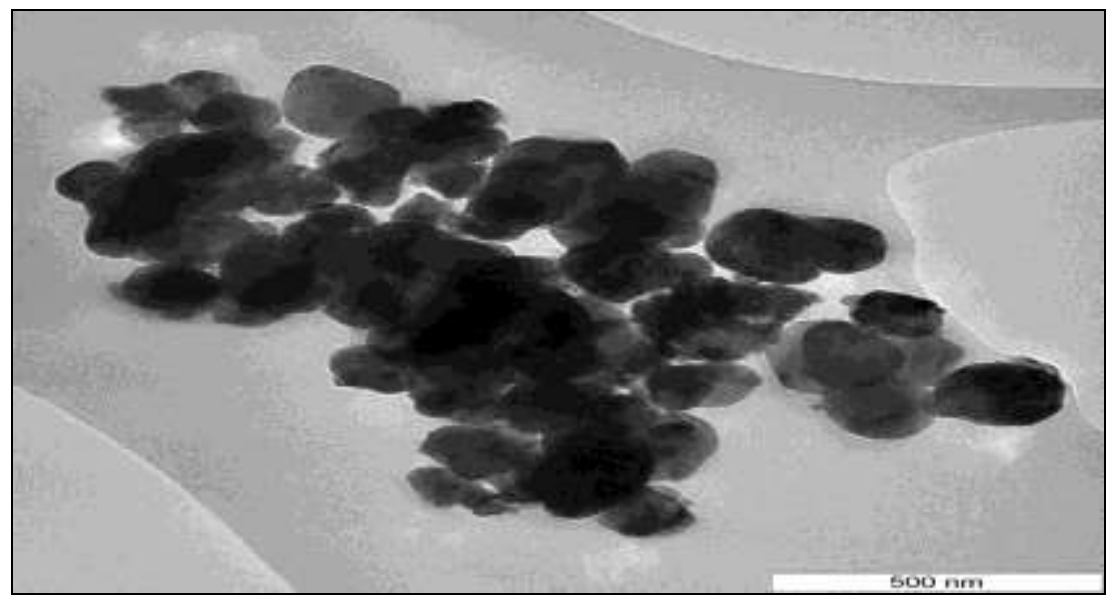

Figure 1: TEM micrograph of as-milled powder after $12 \mathrm{~h}$ of milling 
Figure 2 shows typically the room-temperature XRD patterns of samples sintered at various temperatures ranging from $500{ }^{\circ} \mathrm{C}$ to $1400{ }^{\circ} \mathrm{C}$ for $10 \mathrm{~h}$. The evolution of crystalline phase was investigated as a function of sintering temperature. It can be seen there is no presence of $\mathrm{SrTiO}_{3}$ phase in the as-milled powder, only $\mathrm{SrCO}_{3}$ and $\mathrm{TiO}_{2}$ peaks were observed. These same reflection peaks are also present for $500{ }^{\circ} \mathrm{C}, 600{ }^{\circ} \mathrm{C}$ and $700{ }^{\circ} \mathrm{C}$ revealing the existence of strontium carbonate and titanium dioxide peaks. Certainly by $800{ }^{\circ} \mathrm{C}$, the diffraction peaks corresponding to the $\mathrm{SrTiO} 3$ crystalline phase have clearly appeared and become stronger. On the contrary, the intensity of $\mathrm{TiO}_{2}$ and $\mathrm{SrCO}_{3}$ peaks become weaker. However, these impurities further react with increasing sintering temperature to yield the pure $\mathrm{SrTiO}_{3}$ perovskite phase. When the sintering temperature reached $900{ }^{\circ} \mathrm{C}$, the patterns show well-defined peaks, indicating the crystalline and phase formation of the synthesized compounds. When the sintering temperatures increases further, the intensity keeps increasing indicating progressive improvement in crystallinity of the materials. All the XRD peaks become sharper and narrower with increase in temperatures. Increasing of sintering temperature to $1400{ }^{\circ} \mathrm{C}$ led to the formation of new phase which is $\mathrm{Sr}_{3} \mathrm{Ti}_{2} \mathrm{O}_{7}$. All the diffraction peaks can be indexed to a cubic perovskite structure of $\mathrm{SrTiO}_{3}$ with lattice constant, $\mathrm{a}=3.90 \AA$, which is in good agreement with the literature value. Comparing these patterns, it was found that they belong to a cubic structure with a space group $\mathrm{Pm} 3 \mathrm{~m}$. In summary, the $\mathrm{SrTiO}_{3}$ phase without impurities can only be acquired for the $900^{\circ} \mathrm{C}$-sintered sample.

From this point of view, good $\mathrm{SrTiO}_{3}$ have been produced at a much lower reaction temperature compared to those prepared by conventional solid state method [15]. This is due to higher reactivity between the finer-sized particles yielded by the mechanical alloying technique. Based on the XRD patterns, it was observed that the formation of crystalline $\mathrm{SrTiO}_{3}$ is not possible by milling alone or by using sintering temperatures that are too low. This might occur due to the insufficient energy imparted by the collision of the milling media to the starting powders causing incapability to increase the reactivity between the particles. Other than that, low sintering temperature will provide inadequate energy for the stimulation of the reaction between the particles.

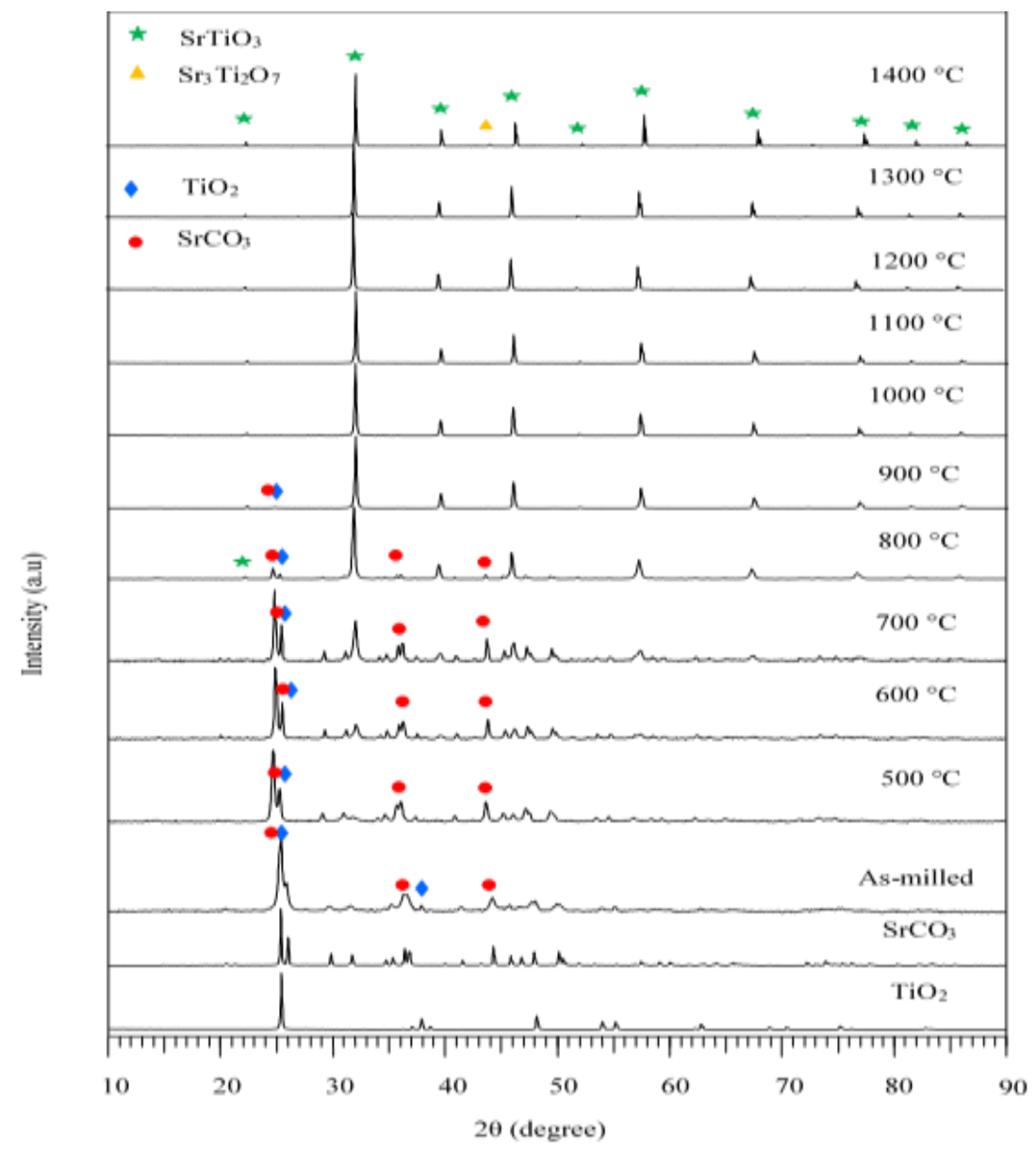

Figure 2: XRD patterns of $\mathrm{SrTiO} 3$ samples showing the structural evolution as a function of the sintering temperatures 
The determination of density and porosity showed that the increase in the sintering temperature has increased the density, reduced the porosity and enhanced the grain size. The data of measured density, theoretical density and porosity sintered at various temperatures are tabulated in Table 1 . The results reveal increasing trend of the density with sintering temperature with the highest density being $5.27 \mathrm{~g} / \mathrm{cm}^{3}$ at $1400{ }^{\circ} \mathrm{C}$ and the lowest density is $4.22 \mathrm{~g} / \mathrm{cm}^{3}$ at $500{ }^{\circ} \mathrm{C}$. The increase in density can be understood by looking at the starting powder with fine particle size due to the alloying process which results in a higher surface energy for a compact. This gives a higher driving force for the crystallite growth and densification. The percentage of porosity decreases linearly from $12.41 \%$ for the sintered sample at $500{ }^{\circ} \mathrm{C}$ to $1.08 \%$ for the sample sintered at $1400^{\circ} \mathrm{C}$. The density and porosity as a function of sintering temperature for all ceramics is plotted in Figure 3

Table 1: Physical properties of $\mathrm{SrTiO}_{3}$ ceramics at different sintering temperatures

\begin{tabular}{|c|c|c|c|c|}
\hline $\begin{array}{c}\text { Sintering } \\
\text { Temperatures }\left({ }^{\circ} \mathrm{C}\right)\end{array}$ & $\begin{array}{c}\text { Measured } \\
\text { density, } \rho_{\exp } \\
\left(\mathrm{g} / \mathrm{cm}^{\mathbf{3}}\right)\end{array}$ & $\begin{array}{c}\text { Theoretical } \\
\text { Density, } \rho_{\text {th }} \\
\left(\mathrm{g} / \mathrm{cm}^{3}\right)\end{array}$ & $\begin{array}{l}\text { Porosity } \\
(\%)\end{array}$ & $\begin{array}{r}\text { Average } \\
\text { Grain Sizes } \\
( \pm 0.1 \mathrm{~nm})\end{array}$ \\
\hline 500 & 4.22 & 4.82 & 12.41 & 126.5 \\
\hline 600 & 4.28 & 4.94 & 13.40 & 146.9 \\
\hline 700 & 4.36 & 4.89 & 10.83 & 148.8 \\
\hline 800 & 4.47 & 4.84 & 7.66 & 179.9 \\
\hline 900 & 4.59 & 4.89 & 6.21 & 188.9 \\
\hline 1000 & 4.68 & 4.90 & 4.41 & 233.9 \\
\hline 1100 & 4.76 & 4.90 & 2.88 & 267.6 \\
\hline 1200 & 4.82 & 4.92 & 2.01 & 355.0 \\
\hline 1300 & 4.86 & 4.93 & 1.38 & 559.6 \\
\hline 1400 & 5.27 & 5.33 & 1.08 & \\
\hline
\end{tabular}

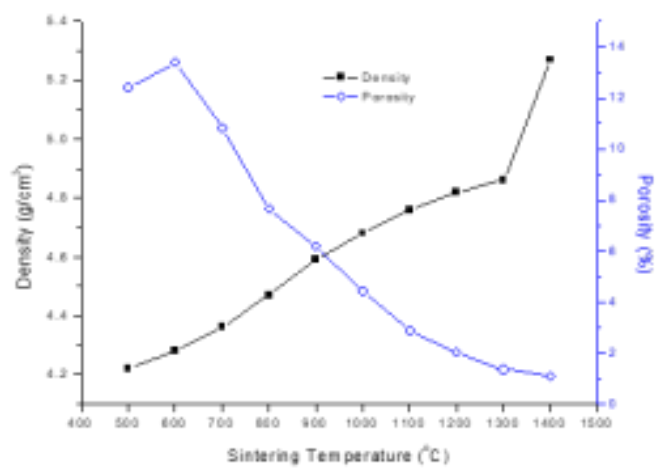

Figure 3: Variation of density and porosity as a function of sintering temperature

The corresponding microstructure and grain size distribution of $\mathrm{SrTiO}_{3}$ samples prepared at different sintering temperatures are shown in Figure 4. Obviously, the sintering temperature has a significant effect on the grain growth or size distribution of the samples. The average grain size was measured over 200 grains by the linear intercept method and increases with increasing sintering temperatures from $\sim 120 \mathrm{~nm}$ to $600 \mathrm{~nm}$, and this increase indicates the microstructural evolution of the samples. The images of the sintered sample at $500{ }^{\circ} \mathrm{C}, 600{ }^{\circ} \mathrm{C}$ and $700{ }^{\circ} \mathrm{C}$ (Figures 4 (a), (b) and (c)) showed strongly agglomerated non-uniform particles with neck formation and coalescence between the nanoparticles, revealing that the samples exhibit an initial stage of sintering. Figures 4(d), (e) and (f) which represent sintering temperatures of $800{ }^{\circ} \mathrm{C}, 900{ }^{\circ} \mathrm{C}$ and $1000{ }^{\circ} \mathrm{C}$ respectively showed the intermediate stage of sintering where particles moves closer leading to shrinkage of the sample. Porous pellets with fine grains were formed at $1100{ }^{\circ} \mathrm{C}$. Referring to the final stage of the sintering process, Figure 4 (h), (i) and (j), grain growth takes place, the pores broke up and form closed spherical bubbles. As for the $1400^{\circ} \mathrm{C}$-sintered sample, the samples show large cracks, the lower densities of impurities $\mathrm{Sr}_{3} \mathrm{Ti}_{2} \mathrm{O}_{7}$ can lead to a volume expansion at the elevated sintering temperature. From this micrograph, formation of pores at the beginning of the sintering process can be observed and it decreased with increased temperature. The average grain size of $\mathrm{SrTiO}_{3}$ samples sintered at various temperatures is tabulated in Table 1. The increases of average grain sizes with increasing sintering temperatures indicates the microstructural evolution of the samples. 


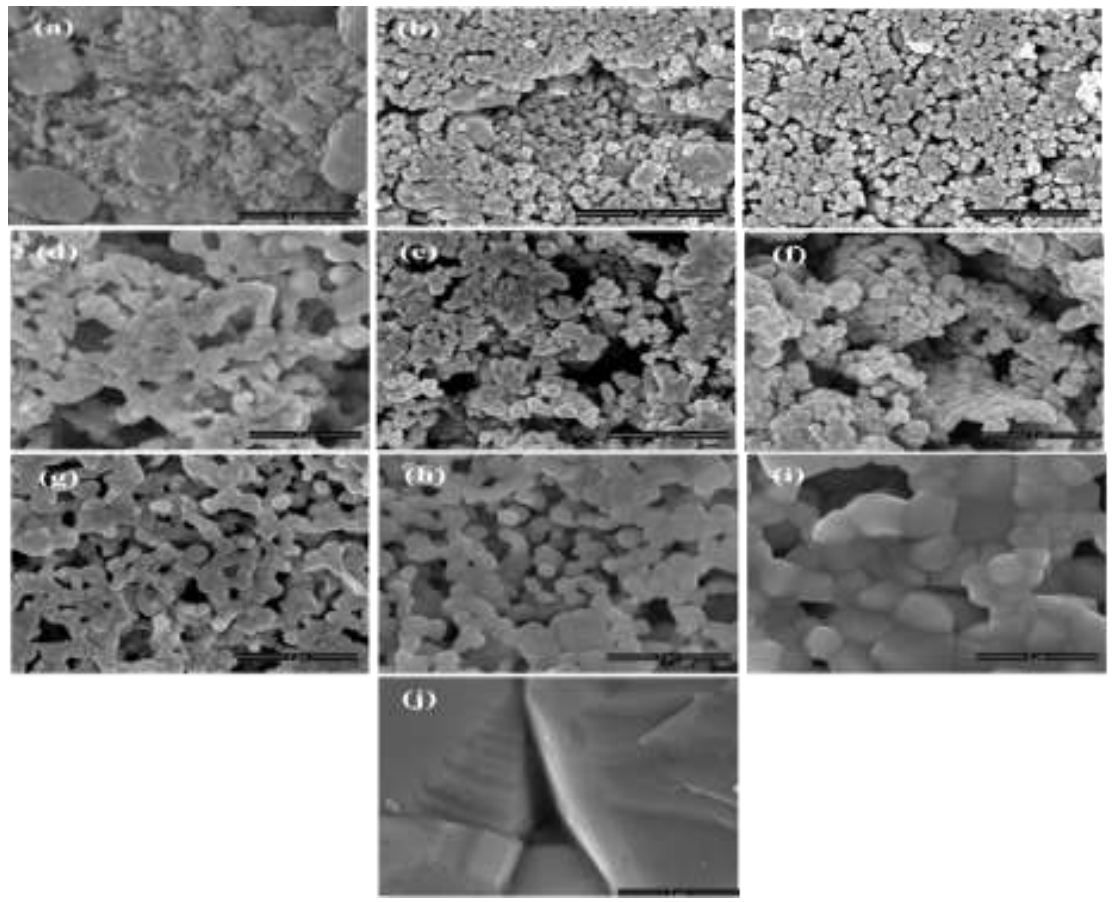

Figure 4: FeSEM images on the surface of $\mathrm{SrTiO}_{3}$ pellets sintered at $500{ }^{\circ} \mathrm{C}-1400{ }^{\circ} \mathrm{C}$ for 12 hours

\subsection{Complex Permittivity}

The dielectric properties of ceramic system were studied in the frequency range from $10 \mathrm{~Hz}$ to $1 \mathrm{MHz}$ at room temperature. Figure 5 shows the variation of the dielectric permittivity components; and as a function of frequency for all the samples and these are tabulated in Table 2. In general, a decrease in the dielectric constant was observed with an increase in frequency. The decrease is rapid at lower frequency and slower and stable at higher frequency. High value of at low frequency is explained on the basis of space charge polarization due to inhomogeneous dielectric structure which is caused by the movement of charge carriers trapped at the interfacial region. The inhomogeneities in the present system are impurities, porosity and grain structure. A decrease in the dielectric constant with increasing frequency is expected in most dielectric materials due to the dielectric relaxation, as the speed of dipole rotation at high frequency is insufficient to match the shift in the applied AC bias [16-18]. At high frequencies, the dominant mechanism contributing to the dielectric constant is the dipolar polarization under the influence of alternating current. This leads to a decrease in the dielectric constant with increase in frequency. The frequency of hopping between ions could not follow the frequency of the applied field and hence it lags behind, therefore the dielectric constant is reduced at higher frequencies [19].


Figure 5 Variation of the dielectric properties, $\varepsilon_{\mathrm{r}}{ }^{\prime}$ and $\tan \delta$ at room temperature as a function of frequency for $\mathrm{SrTiO}_{3}$ ceramic at different sintering temperatures. 
Figure 6 shows the sintering temperature and grain size dependence of the dielectric constant at $1 \mathrm{MHz}^{\mathrm{for}} \mathrm{SrTiO}_{3}$ ceramics. From Figure 6(a), it is observed that the dielectric constant increases with increasing sintering temperature. and sintering temperature exhibit a similar trend as that between density and sintering temperature. This phenomenon can be explained by the increase in density and in grain size as the sintering temperature is increased [20-22]. In addition, agglomerations of powders become a resistant for ions to polarize between grains and grain boundaries [15]. Theoretically, dielectric properties are related to ion polarizabilities by the Clausius-Mosotti equation [23]. It is believed that polarization of ions in grains may increase with grain necking growth.

Figure 5(6) for sintering temperatures $500{ }^{\circ} \mathrm{C}, 600{ }^{\circ} \mathrm{C}$ and $700{ }^{\circ} \mathrm{C}$ shows that the dielectric constant is small. This can be related with the crystalline phase. As we refer to Figure 2, the amorphous phase is still large which means that there were low crystalline phases. Figure 6(b) also shows a sudden rise in the dielectric constant from 67.3 to 130.1 for sintering temperatures $700^{\circ} \mathrm{C}$ to $800^{\circ} \mathrm{C}$ even though the increment in the grain size is only $31 \mathrm{~nm}$. This is due to the formation of $\mathrm{SrTiO}_{3}$ phase at this stage and as is well known that the dielectric constant of $\mathrm{SrTiO}_{3}$ is much bigger than $\mathrm{SrCO}_{3}$ and $\mathrm{TiO}_{2}$. Further increase from $800^{\circ} \mathrm{C}$ to $900^{\circ} \mathrm{C}$ resulted in a steeper increase in the dielectric constant. This corresponds to the XRD data which is a shift from mixed phase to pure phase of $\mathrm{SrTiO}_{3}$. A steady increase in the dielectric constant is observed at the higher sintering temperature. This suggested that there is development in the grain size which improves the crystallinity thus resulting in a higher dielectric value from $1000^{\circ} \mathrm{C}$ to $1400^{\circ} \mathrm{C}$ which is reflected in Table 2. These prove the influence of microstructure-evolution changes on dielectric properties in strontium titanate

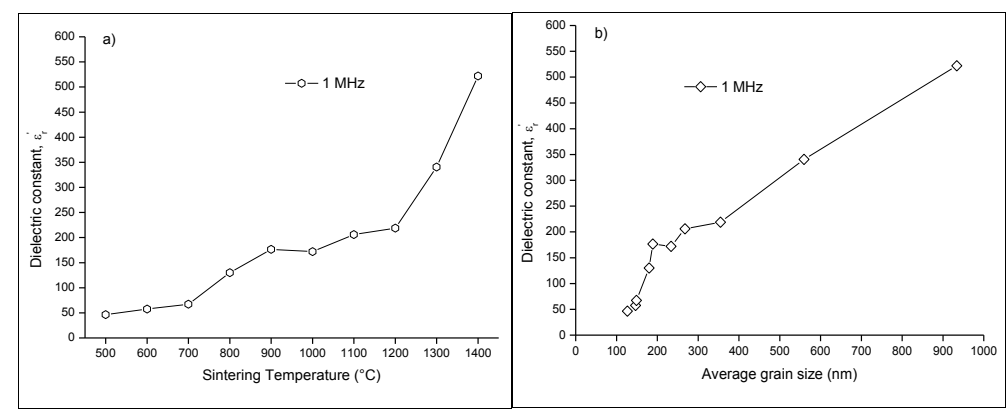

Figure 5: Dielectric constant as a function of (a) sintering temperature (b) grain size for $\mathrm{SrTiO}_{3}$ at $1 \mathrm{MHz}$

Table 2: Dielectric constant and loss tangent of ST at different sintering temperatures measured at room temperature.

\begin{tabular}{|c|c|c|c|c|c|c|c|c|}
\hline \multirow{2}{*}{$\begin{array}{c}\text { Sintering } \\
\text { Temperature } \\
\left({ }^{\circ} \mathrm{C}\right)\end{array}$} & \multicolumn{4}{|c|}{ Dielectric constant, $\varepsilon_{r}$} & \multicolumn{4}{|c|}{$\tan \delta$} \\
\hline & $1 \mathrm{kHz}$ & $10 \mathrm{kHz}$ & $100 \mathrm{kHz}$ & $1 \mathrm{MHz}$ & $1 \mathrm{kHz}$ & $10 \mathrm{kHz}$ & $100 \mathrm{kHz}$ & $1 \mathrm{MHz}$ \\
\hline $500^{\circ} \mathrm{C}$ & 93.808 & 56.330 & 49.753 & 46.645 & 0.611 & 0.244 & 0.069 & 0.031 \\
\hline $600^{\circ} \mathrm{C}$ & 104.540 & 66.255 & 59.363 & 57.615 & 0.749 & 0.229 & 0.064 & 0.019 \\
\hline $7^{700}{ }^{\circ} \mathrm{C}$ & 82.891 & 70.770 & 68.324 & 67.367 & 0.337 & 0.077 & 0.021 & 0.017 \\
\hline $800^{\circ} \mathrm{C}$ & 142.799 & 133.213 & 130.605 & 130.176 & 0.133 & 0.032 & 0.009 & 0.009 \\
\hline $900^{\circ} \mathrm{C}$ & 224.632 & 186.733 & 178.903 & 176.407 & 0.277 & 0.080 & 0.025 & 0.008 \\
\hline $1000^{\circ} \mathrm{C}$ & 195.506 & 175.286 & 172.460 & 171.972 & 0.214 & 0.046 & 0.009 & 0.005 \\
\hline $1100^{\circ} \mathrm{C}$ & 208.274 & 206.267 & 205.696 & 205.918 & 0.013 & 0.003 & 0.001 & 0.004 \\
\hline $1200^{\circ} \mathrm{C}$ & 225.530 & 220.273 & 218.986 & 218.883 & 0.055 & 0.013 & 0.004 & 0.002 \\
\hline $1300^{\circ} \mathrm{C}$ & 339.386 & 340.006 & 338.955 & 340.646 & 0.004 & 0.001 & 0.001 & 0.005 \\
\hline $1400^{\circ} \mathrm{C}$ & 519.239 & 518.307 & 517.603 & 522.126 & 0.008 & 0.001 & 0.002 & 0.018 \\
\hline
\end{tabular}

\section{CONCLUSION}

Nanosized $\mathrm{SrTiO}_{3}$ of $80 \mathrm{~nm}$ was successfully synthesized using mechanical alloying method after $12 \mathrm{~h}$ of high-energy milling with the formation of the perovskite $\mathrm{SrTiO}_{3}$ phase at $900^{\circ} \mathrm{C}$ which is at a lower temperature compared to the solid state method. The parallel effects of sintering temperature on morphological properties and dielectric properties in SrTiO3 have been investigated. Structural and morphology of the particles were systematically analyzed employing various analytical tools such as TEM, XRD and SEM where it revealed the microstructure and the reduction of grain size with high energy ball milling. SEM micrographs and the process of grain growth at different temperatures indicated the 
microstructural evolution of the sample. The dielectric constant of the sintered samples at $1 \mathrm{MHz}$ increased from 46.6 to 522.1 as the sintering temperature increased from $500^{\circ} \mathrm{C}$ to $1400^{\circ} \mathrm{C}$. There are two polarization mechanisms in $\mathrm{SrTiO}_{3}$ which are interfacial and dipolar polarizations. By correlating the dielectric properties and the microstructural evolution characteristics, there are developments in the grain size which improve the crystallinity thus reflecting in a higher dielectric constant.

\section{ACKNOWLEDGEMENT}

This work was financially supported by MyBrain 15 Scholarship. The authors gratefully acknowledge Institute of Advanced Technology (ITMA), and Faculty of Science, Universiti Putra Malaysia for the facilities provided

\section{REFERENCES}

[1] V. Brizé, et al., "Grain size effects on the dielectric constant of $\mathrm{CaCu}_{3} \mathrm{Ti}_{4} \mathrm{O}_{12}$ ceramics", Materials Science and Engineering B, 129 135-138 (2006).

[2] Chunhong $\mathrm{Mu}$, et al., Microstructural and Dielectric Properties of $\mathrm{SrTiO}_{3}$-doped $\mathrm{CaCu}_{3} \mathrm{Ti}_{4} \mathrm{O}_{12}$ Ceramics", Materials Science Forum, 687 321-326 (2011)

[3] C. N. R. Rao and John M. Thomas, "Intergrowth structures: the chemistry of solid-solid interfaces", Acc. Chem. Res 18 113-119 (1985).

[4] Rao C N R, Gopalakrishnan J and Vidyasagar K, Indian J. Chem. A23 265(1984)

[5] Anderson MT and Poeppelmeier K R., "Lanthanum copper tin oxide $\left(\mathrm{La}_{2} \mathrm{CuSnO}_{6}\right)$ : a new perovskite-related compound with an unusual arrangement of B cations", Chem. Mater 3 476-482 (1991).

[6] Anderson MT, et al. B-cation arrangements in double perovskites", Prog. Solid State Chem 22 197-233 (1993).

[7] A. Tkach, et al., "Synthesis by sol-gel and characterization of strontium titanate powder", Key Engineering Materials 230-232 40-43 (2002)

[8] C. Suryanarayana, F. H. Froes, "The structure and mechanical properties of metallic nanocrystals" Metallurgical Transactions A 23 1071-1081 (1992).

[9] C. Suryanarayana, "Mechanical alloying and milling”, Progress in Materials Science, 46, 1-184 (2011)

[10] C. Suryanarayana, "Recent Developments in Mechanical Alloying”, Rev.Adv. Mater.Sci. 18203-211 (2008)

[11] Quirk, J. F., "Factors affecting sinterability of oxide powders: BeO and MgO" J. Am. Ceram. Soc. 42 4 178-181 (1959)

[12] Gupta, T. K. and R.L. Coble, "Sintering of ZnO: Densification and grain growth” J. Am. Ceram. Soc., $519521-525$ (1968).

[13] Kang, S.-J.L., Kim, K.-H. \& Yoon, D.N. "Densification and Shrinkage during Liquid Phase Sintering”, J. Am. Ceram. Soc., 742 425-427 (1991).

[14] Huang, C.-L. \& Tsai, J.-T. "Effects of Sintering Temperature on CaO- $\mathrm{Li}_{2} \mathrm{OSm}_{2} \mathrm{O}_{3}-\mathrm{TiO}_{2}$ Microwave Dielectric Ceramic" Proc. Natl. Sci. Counc. ROC (A), 255 317-321 (2001).

[15] Leow Chun Yan, et al, "Effect of Sintering Temperatures on the Microstructure and Dielectric Properties of SrTiO3” World Applied Sciences Journal, 14 (7), 1091-1094. .(2011).

[16] Chenari H. Mahmoudi, Ali Hassanzadeh, M.M. Golzan, H. Sedghi, M. Talebian , "Frequency dependence of ultrahigh dielectric constant of novel synthesized SnO2 nanoparticles thick films" Current Applied Physics 11409 413 (2011)

[17] Venkataraman, B. H., K.B.R. Varma "Frequency-dependent dielectric characteristics of ferroelectric $\mathrm{SrBi}_{2} \mathrm{Nb}_{2} \mathrm{O}_{9}$ ceramics" Solid State Ionics 167 197-202 (2004)

[18] Ravinder D., G. Ranga Mohan, Prankishan, Nitendarkishan, D.R. Sagar, "High frequency dielectric behaviour of aluminium-substituted lithium ferrites" Materials Letters, 44 256-260(2000)

[19] Kumar, G. B. and Buddhudu, S. "Optical, thermal and dielectric properties of $\mathrm{Bi}_{4}\left(\mathrm{TiO}_{4}\right)$ ceramic powders" Ceram. Int., 36, 1857-1861. (2010)

[20] Wu A et al. "Sol-gel preparation of lead zirconate titanate powders and ceramics: Effect of alkoxide stabilizers and lead precursors", J Am Ceram Soc, 83: 1379-1385. (2000)

[21] Shaikh PA, Kolekar YD, "Study of microstructural, electrical and dielectric properties of perovskite (0.7) PMN(0.3) PT ferroelectric at different sintering temperatures", J Anal Appl Pyrol, 93 41-46 (2012).

[22] Prasad KVR, Raju AR, Varma KBR. "Grain size effects on the dielectric properties of ferroelectric $\mathrm{Bi}_{2} \mathrm{VO}_{5.5}$ ceramics". J Mater Sci, 29: 2691-2696 (1994).

[23] Shannon RD. "Dielectric polarizabilities of ions in oxides and fluorides” J Appl Phys 73: 348-366. (1993). 